\title{
Quasiparticles in the Superconducting State of $\mathrm{Bi}_{2} \mathrm{Sr}_{2} \mathrm{CaCu}_{2} \mathrm{O}_{8+\delta}$
}

\author{
A. Kaminski, ${ }^{1,2}$ J. Mesot, ${ }^{2}$ H. Fretwell, ${ }^{1}$ J. C. Campuzano, ${ }^{1,2}$ M. R. Norman, ${ }^{2}$ M. Randeria, ${ }^{3}$ H. Ding, ${ }^{4}$ T. Sato, ${ }^{5}$ \\ T. Takahashi, ${ }^{5}$ T. Mochiku, ${ }^{6}$ K. Kadowaki, ${ }^{7}$ and H. Hoechst ${ }^{8}$ \\ ${ }^{1}$ Department of Physics, University of Illinois at Chicago, Chicago, Illinois 60607 \\ ${ }^{2}$ Materials Science Division, Argonne National Laboratory, Argonne, Illinois 60439 \\ ${ }^{3}$ Tata Institute of Fundamental Research, Mumbai 400005, India \\ ${ }^{4}$ Department of Physics, Boston College, Chestnut Hill, Massachusetts 02467 \\ ${ }^{5}$ Department of Physics, Tohoku University, 980-8578 Sendai, Japan \\ ${ }^{6}$ National Research Institute for Metals, Sengen, Tsukuba, Ibaraki 305, Japan \\ ${ }^{7}$ Institute of Materials Science, University of Tsukuba, Ibaraki 305, Japan \\ ${ }^{8}$ Synchrotron Radiation Center, Stoughton, Wisconsin 53589 \\ (Received 27 April 1999; revised manuscript received 7 October 1999)
}

\begin{abstract}
Recent improvements in momentum resolution lead to qualitatively new angle-resolved photoemission spectroscopy results on the spectra of $\mathrm{Bi}_{2} \mathrm{Sr}_{2} \mathrm{CaCu}_{2} \mathrm{O}_{8+\delta}$ (Bi2212) along the $(\pi, \pi)$ direction, where there is a node in the superconducting gap. We now see the intrinsic line shape, which indicates the presence of true quasiparticles at all Fermi momenta in the superconducting state, and lack thereof in the normal state. The region of momentum space probed here is relevant for charge transport, motivating a comparison of our results to conductivity measurements by infrared reflectivity.
\end{abstract}

PACS numbers: 74.25.Jb, 71.18.+y, 74.72.Hs, 79.60.Bm

Landau's concept of the Fermi liquid [1] underlies much of our present theoretical understanding of electron dynamics in crystalline solids. Landau was able to demonstrate that, even though the electrons interact strongly with one another, one can still describe the low temperature properties of metals in terms of "quasiparticle" excitations, which are bare electrons dressed by the medium in which they move. But we now have materials, such as the high temperature superconductors (HTSCs), and other low dimensional systems, where it is becoming increasingly difficult to reconcile experimental results with the expectations of Fermi liquid theory $[2,3]$.

Clearly, if the concept of quasiparticles is to be useful, they must live long enough to be considered as independent entities. In fact, in a Fermi liquid, the quasiparticles at the Fermi momentum, $k_{F}$, have (at zero temperature) an infinite lifetime at zero excitation energy - the Fermi energy, $E_{F}$ - with their lifetime decreasing quadratically with excitation energy [1]. If one were to measure the spectral function of the electrons at $k_{F}$, one would observe a broad feature, corresponding to the incoherent part of the electron, with a sharp peak at $E_{F}$ with spectral weight $z$, the quasiparticle component. It is now well established by angle-resolved photoemission spectroscopy (ARPES) measurements that, despite the existence of a Fermi surface in momentum space, there are no quasiparticles in the normal state of optimally doped or slightly overdoped HTSCs near the $(\pi, 0)$ point of the Brillouin zone (see inset of Fig. 1) [4,5]. Below $T_{c}$, the superconducting gap is maximal here (at the antinode point $A$ ) and sharp quasiparticle peaks are observed [6]. This statement can be made since the energy dispersion of the electronic states in this region of the zone is very weak, and therefore the measured spectra are not artificially broadened by the finite acceptance angle (momentum window) of the detector.

Along the zone diagonal, however, the intrinsic line shape is unknown, both in the normal and superconducting states, because the spectra near point $N$ (at the Fermi surface along the diagonal; see inset of Fig. 1) are significantly broadened by the momentum window given the highly dispersive nature $(1.6 \mathrm{eV} \AA)$ of the states in this region. This is reinforced by lack of the observation of any temperature dependence of the spectra at point $N$ in sharp contrast to what is observed at point $A$. In this work, a large improvement in experimental momentum resolution allows us to determine the intrinsic line shape at $N$ for the first time. The significance of these observations stems from the fact that this region of the zone dominates many of the bulk properties of cuprate superconductors, and it is very important to know if and when quasiparticles exist. Above $T_{c}$, the charge and thermal transport are dominated by these states because of their rapid dispersion (large Fermi velocity). Below $T_{c}$, the superconducting energy gap vanishes at the nodal point $N$, and low energy excitations in its neighborhood dominate the $T$ dependence of various properties, e.g., the superfluid density [7] and thermal Hall conductivity [8]. Moreover, there has been a gamut of opinions concerning the nature of the electronic states near the node, ranging from the cold spot model [9] where quasiparticles are assumed to be present even above $T_{c}$, and the stripes model [10], where quasiparticles do not exist even below $T_{c}$.

Measurements were carried out at the SRC, Wisconsin, on a $4 \mathrm{~m}$ NIM undulator beam line (resolving power of $10^{4}$ at $10^{12}$ photons/sec) as well as a plane grating monochromator beam line. We used a Scienta SES 200 analyzer in angle resolved mode. The angular resolution and spacing 

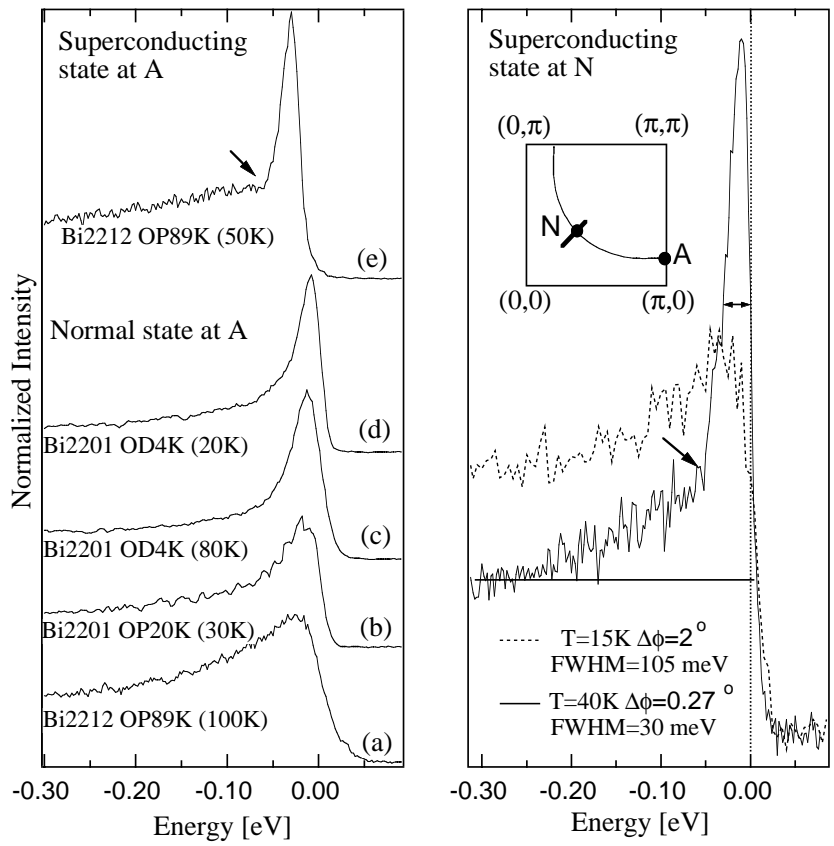

FIG. 1. Left panel: ARPES spectra at point $A$ of the zone (inset), with spectra labeled by doping (OD for overdoped, OP for optimal doped) and onset $T_{c}$. The temperature is given in parenthesis. Right panel: ARPES spectra of Bi2212 $\left(T_{c}=\right.$ $89 \mathrm{~K}$ ) in the superconducting state at point $N$ for two values of the resolution. We define FWHM of the peak by the horizontal arrow assuming that peak height is measured from a horizontal line drawn at the background level. Note the break in the high resolution spectrum marked by an arrow.

of EDCs is $0.0097 \AA^{-1}$ at $22 \mathrm{eV}$ as calibrated by measuring the superlattice wave vector. The energy resolution was $16 \mathrm{meV}$ (FWHM). Samples were mounted with the $\Gamma X$ direction parallel to the photon polarization, except for Fig. 2 right panel and Fig. 1 left panel, where samples were aligned along $\Gamma \bar{M}$. ARPES gives direct information about the momentum and energy dependence of the lifetime of electrons. In quasi-2D materials, the ARPES signal is given by [6] $I(\omega)=I_{\mathbf{k}} f(\omega) A(\mathbf{k}, \omega)$, convolved with the energy resolution and momentum aperture of the detector. Here $I_{\mathbf{k}}$ is the dipole matrix element between initial and final states, $f(\omega)$ the Fermi function, and $A(\mathbf{k}, \omega)$ the spectral function.

Curve (a) in the left panel of Fig. 1 shows an ARPES spectrum in the normal state at $100 \mathrm{~K}$ for an optimally doped $\mathrm{Bi} 2212$ sample with $T_{c}=89 \mathrm{~K}$. In principle, at the Fermi surface $\left(k=k_{F}\right), A(\mathbf{k}, \omega)$ should have a peak centered at zero binding energy. Since ARPES measures only the occupied part of $A$, the spectral peak is cut off by the Fermi function, and thus its maximum is displaced to higher energy. Therefore an estimate of its full width-half maximum (FWHM) can be obtained by doubling the measured one, yielding a value of $\sim 200 \mathrm{MeV}$. Although quasiparticles are expected to appear only at low temperatures, we note this width is of the order of $2000 \mathrm{~K}$, well over an order of magnitude larger than the temperature,
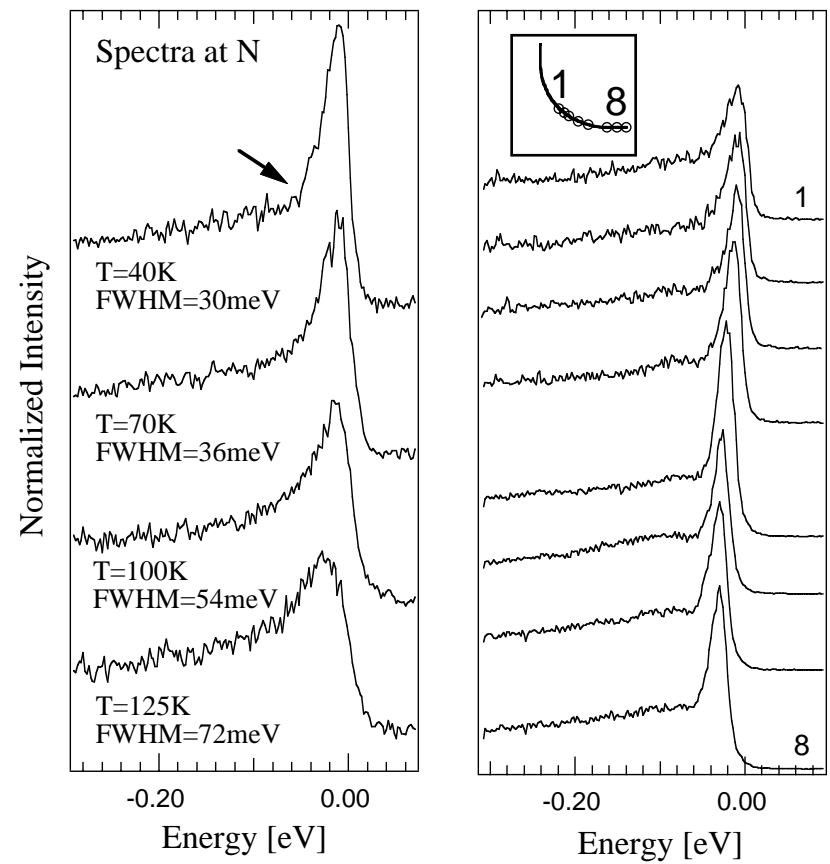

FIG. 2. Left panel: Temperature dependence of the spectra at point $N$ (inset of Fig. 1) for a Bi2212 sample $\left(T_{c}=89 \mathrm{~K}\right)$. A break in the line shape of the spectra at $40 \mathrm{~K}$ is indicated by an arrow. Right panel: Comparison of the superconducting state spectra $(T=40 \mathrm{~K})$ at various points along the Fermi surface as shown in the inset.

indicating that thermal broadening alone cannot be responsible for the large peak width.

We can confirm that large widths are intrinsic to optimally doped cuprates by examining the spectral function of the single $\mathrm{CuO}$ layer compound Bi2201, which has a lower $T_{c}$, and therefore a normal state accessible at a lower temperature. In curve (b) of Fig. 1 we plot ARPES data for the slightly overdoped Bi2201 compound $\mathrm{Bi}_{1.6} \mathrm{~Pb}_{0.4} \mathrm{Sr}_{2} \mathrm{CuO}_{6}\left(T_{c}=20 \mathrm{~K}\right)$. Even though the normal state data are now taken at $30 \mathrm{~K}$, the spectral width has narrowed to only a FWHM of $100 \mathrm{meV}$, and so does not exhibit a quasiparticle peak. We therefore conclude that quasiparticles do not appear in the normal state of optimally doped compounds at point $A$, even at low temperature. As there is some evidence from transport that more Fermi liquidlike behavior develops for heavily overdoped materials, the question arises whether ARPES sees evidence for normal state quasiparticles in that case. Curve (d) of Fig. 1 shows a spectrum for a highly overdoped Bi2201 sample $\left(T_{c}=4 \mathrm{~K}\right)$ at $20 \mathrm{~K}$. Indeed, the spectral peak is much narrower than the optimally doped sample, consistent with more Fermi liquidlike behavior. It can be seen that the broadening of the peak in the optimally doped sample is not of thermal origin by comparing it to the overdoped sample at a higher temperature, shown in curve (c) of Fig. 1. The width of the peak of the overdoped sample at $80 \mathrm{~K}$ is in fact narrower than that of the optimally doped sample at $30 \mathrm{~K}$. 
Although the normal state of optimally doped cuprates does not exhibit them, quasiparticles do appear in the superconducting state at point $A$. Curve (e) in Fig. 1 shows a spectrum in the superconducting state, where a near resolution limited peak appears. In addition, a break clearly separates the coherent quasiparticle part of the spectral function from the incoherent part, as indicated by the arrow [11]. A significant point is that in the vicinity of the $A$ point, the break in the spectra appears exactly at $T_{c}$ [12]. This is thought to be due to the onset of superconductivity, which leads to a reduction of the scattering rate of electrons over an energy range of order 2-3 times the maximum superconducting gap [13], thus allowing quasiparticles to exist.

It is now well established that the HTSCs are $d$-wave superconductors with points $N$ characterized by nodes in the energy gap [4,5]. We now ask the question of whether quasiparticles exist at these particular points which exhibit gapless excitations. So far, it has not been possible to address this question because of resolution issues. For example, the dashed curve in the right panel of Fig. 1 shows the spectrum obtained at $N$ with the momentum resolution of all previously published work, deep in the superconducting state $(T=15 \mathrm{~K})$. Although this peak has been called a "quasiparticle peak" in the literature, there is no direct evidence for this in the raw data. The spectrum is as broad as the normal state spectrum of curve (a) in Fig. 1, but for a different reason. This is the direction in momentum space of largest dispersion, and the finite momentum window $\delta k$ of the analyzer broadens the peaks as $\delta E=\hbar v_{F} \delta k$, where $v_{F}$ is the Fermi velocity. From the observed dispersion, this momentum broadening is of the order of $100 \mathrm{meV}$.

Now, with a 32-fold improvement in momentum resolution from the Scienta detector (8 times along the direction normal to the Fermi surface, and 4 times in the transverse direction), we show in the solid curve of the right panel of Fig. 1 the qualitatively new result that there are true quasiparticles at the nodes of the $d$-wave superconducting state [14]. Although these data were obtained in the superconducting state, because they are at the node of the $d$-wave state, they are in fact gapless. A break separating the coherent from the incoherent part of the spectral function is visible in the line shape as indicated by an arrow. But the converse is also true: there are no signs of quasiparticles in the normal state. Figure 2 (left panel) shows the temperature dependence of the line shape at point $N$. We note that there is a distinct change in line shape due to the appearance of quasiparticles in the optimally doped sample. In the normal state, the trailing edge of the spectral peak smoothly evolves into an incoherent tail going to high binding energy. As the temperature is lowered below $T_{c}$, a break develops which separates the coherent quasiparticle peak from the incoherent tail. It is important to note that quasiparticles exist all along the Fermi surface (Fig. 2b).
In Fig. 3, we plot the momentum dependence of the line shape along the zone diagonal (at $45^{\circ}$ to the $\mathrm{Cu}-\mathrm{O}$ bond direction) in the normal $(125 \mathrm{~K})$ and superconducting $(40 \mathrm{~K})$ states for an optimally doped $89 \mathrm{~K}$ sample. We note the sharpening of the spectral peak in the normal state as $k_{F}$ is approached, as observed before [15]. In the superconducting state, we see the new result of a coherent peak along this direction, which exists only in a narrow momentum interval about $k_{F}$. More quantitative information can be obtained by plotting the FWHM of the spectral peak from the raw data as a function of the binding energy of the peak, as shown in Fig. 4. Above $T_{c}$, the FWHM is approximately linear in binding energy (we define the FWHM relative to the horizontal line in the right panel of Fig. 1), with a slope of 0.5. An extrapolation of the linear part to zero binding energy results in an offset of $80 \mathrm{meV}$, about an order of magnitude larger than the temperature. Below $T_{c}$, the FWHM is the same as that in the normal state for binding energies above an energy of about 2-3 times the maximum superconducting gap $\left(\Delta_{\max }=40 \mathrm{MeV}\right)$, but decreases faster than this below, as expected for electron-electron scattering [16]. The

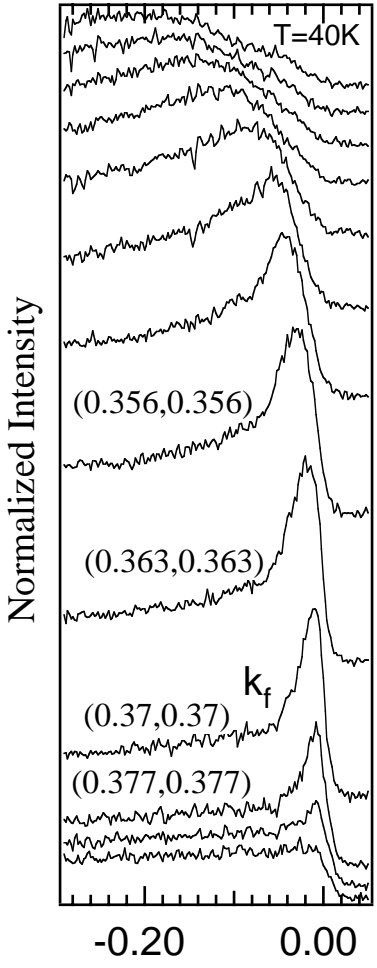

Energy $[\mathrm{eV}]$

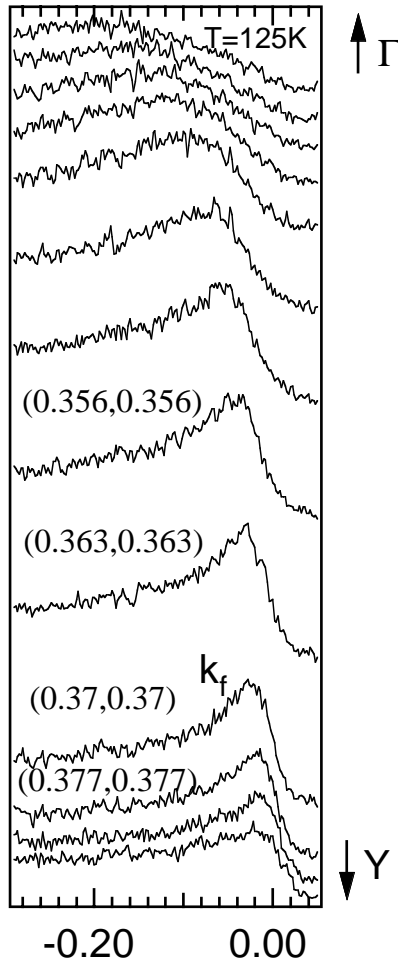

Energy $[\mathrm{eV}]$
FIG. 3. Momentum dependence of the spectra of Bi2212 $\left(T_{c}=89 \mathrm{~K}\right)$ along the $(\pi, \pi)$ direction-indicated by the dark line segment in the inset of Fig. 1 -in the normal $(125 \mathrm{~K})$ and superconducting $(40 \mathrm{~K})$ states. Data were taken at intervals of $0.0097 \AA^{-1}$. The spectrum corresponding to the Fermi momentum is labeled $k_{f}$ and selected spectra are labeled in $\pi / a$ units 


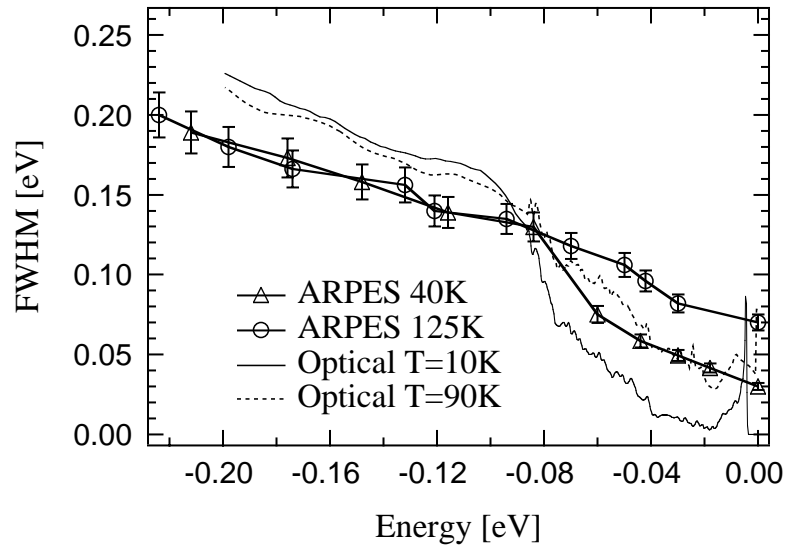

FIG. 4. Full width at half maximum of the spectral peaks in Fig. 3 versus the binding energy of the spectral peak (symbols), and the carrier scattering rate versus energy for Bi2212 $\left(T_{c}=\right.$ $90 \mathrm{~K}$ ) obtained from infrared reflectivity measurements (solid and dashed lines) [17]. The FWHM is defined by the horizontal arrow in the right panel of Fig. 1.

residual offset at zero binding energy is a combination of momentum and energy resolution, and a contribution from the incoherent tail of the spectra (the FWHM of the coherent peak is $30 \mathrm{meV}$, compared to a resolution estimate of $22 \mathrm{meV}$ ).

A detailed comparison of ARPES data to optical conductivity data would require fitting the spectra to a model self-energy, using this self-energy to construct the transport scattering rate, $1 / \tau$, and then averaging $1 / \tau$ over the Brillouin zone with velocity weighting factors [17]. Rather than go through such a complicated procedure, we elect to directly compare the $1 / \tau$ from optical conductivity in Bi2212 to the FWHM versus binding energy (discussed above), which should be a rough measure of $1 / \tau$ versus energy. This is also shown in Fig. 4, where a good match is seen between these two quantities (at low energies, there is a deviation due to the ARPES resolution). We observe that the break in the spectrum near $k_{F}$ separating the coherent peak from the incoherent tail occurs at the same energy, indicating a drop in the imaginary part of the self-energy at the same frequency that optical data see a drop in $1 / \tau$. Finally, we note that the linear energy variation seen by the optical and APRES data is analogous to the linear temperature dependence of the resistivity, ubiquitous in the cuprate superconductors, and is in support of a marginal Fermi liquid phenomenology [3].

In conclusion, we report the first observation of the intrinsic line shape along the zone diagonal, from which we deduce a quasiparticle peak below $T_{c}$ and the absence of such a peak in the normal state. The nonexistence of quasiparticles in the normal state appears to be a general property of cuprate superconductors. Therefore, the formation of quasiparticles must be related to the presence of a superconducting state. A proper description of high temperature superconductivity will arise only after this peculiar phenomenon is understood.

We thank T. Timusk for providing the optical data. This work was supported by the National Science Foundation No. DMR 9624048, and No. DMR 91-20000 through the Science and Technology Center for Superconductivity, the U.S. Department of Energy, Basic Energy Sciences, under Contract No. W-31-109-ENG-38, the CREST of JST, and the Ministry of Education, Science, and Culture of Japan. The Synchrotron Radiation Center is supported by NSF No. DMR 9212658. J.M. is supported by the Swiss National Science Foundation, and M. R. in part by the Indian DST through the Swarnajayanti scheme.

Note added. - After the completion of this work, we became aware of similar work by Valla et al. [18] with somewhat different conclusions.

[1] P. Nozieres, Theory of Interacting Fermi Systems (AddisonWesley, Reading, 1964).

[2] P. W. Anderson, The Theory of Superconductivity in the High $T_{c}$ Cuprates (Princeton University Press, Princeton, New Jersey, 1997).

[3] C. M. Varma et al., Phys. Rev. Lett. 63, 1996 (1989).

[4] Z.-X. Shen and D. S. Dessau, Phys. Rep. 253, 1 (1995).

[5] J. C. Campuzano et al., in The Gap Symmetry and Fluctuations in High- $T_{c}$ Superconductors, edited by J. Bok et al. (Plenum, New York, 1998), p. 229.

[6] M. Randeria et al., Phys. Rev. Lett. 74, 4951 (1995).

[7] W. N. Hardy et al., Phys. Rev. Lett. 70, 3999 (1993).

[8] K. Krishana et al., Phys. Rev. Lett. 75, 3529 (1995). These data imply a long quasiparticle mean free path.

[9] D. Pines, Z. Phys. B 103, 129 (1997); L. B. Ioffe and A. J. Millis, Phys. Rev. B 58, 11631 (1998).

[10] T. Tohyama, S. Nagai, Y. Shibata, and S. Maekawa, Phys. Rev. Lett. 82, 4910 (1999).

[11] The separation of the spectral function into a quasiparticle piece and an incoherent part can be justified from the breakup of the Green's function into corresponding singular and regular parts (Ref. [1]).

[12] M. R. Norman et al., Phys. Rev. B 57, R11 093 (1998).

[13] M. R. Norman et al., Phys. Rev. Lett. 79, 3506 (1997).

[14] Note the node itself is a single point, so it makes a contribution of measure zero to the spectrum.

[15] C. G. Olson et al., Phys. Rev. B 42, 381 (1990).

[16] The reduction in the electron-electron scattering rate below $3 \Delta_{\max }$ was noted in Ref. [1]. For a discussion in the high $T_{c}$ context, see P. Littlewood and C. M. Varma, Phys. Rev. B 46, 405 (1992).

[17] A. V. Puchkov, D. N. Basov, and T. Timusk, J. Phys. Condens. Matter 8, 10049 (1996).

[18] T. Valla et al., Science 285, 2110 (1999). 